\title{
Contrast Enhancement of Intracranial Aneurysms on 3T 3D Black-Blood MRI and Its Relationship to Aneurysm Recurrence following Endovascular Treatment
}

\author{
(D) S. Elsheikh, (DH. Urbach, and (D). Meckel
}

\begin{abstract}
BACKGROUND AND PURPOSE: Endovascular treatment of intracranial aneurysms aims at preventing aneurysm rupture. Contrastenhanced black-blood MR imaging facilitates imaging of the vessel walls and the aneurysmal sac contents and is therefore suitable for studying the healing process. This study aims to describe imaging findings of aneurysmal contrast enhancement following endovascular treatment and its correlation to recurrence and elapsed time since coiling.
\end{abstract}

MATERIALS AND METHODS: Patients undergoing MR imaging follow-up after endovascular treatment of an intracranial aneurysm were included. Contrast-enhanced black-blood TIWI was acquired. Aneurysm wall and intra-aneurysmal enhancement were independently assessed by 2 neuroradiologists and were related to the time elapsed since coiling and the presence of recurrence.

RESULTS: Thirty aneurysms in 30 patients were included. The median time elapsed since treatment was 417 days (interquartile range, 189-1273 days). Aneurysmal contrast enhancement was seen in 24/30 (80\%) aneurysms. Enhancement inside the sac in $55 \%(n=17)$, the wall in $23 \%(n=7)$, at the dome $(n=1)$, or at the base $(n=6)$ was observed. No statistically significant correlation between aneurysmal contrast enhancement and the elapsed time $(P=.83)$ and presence of a recurrence $(P=.184)$ was detected. In $28 / 30$ patients, the images were of adequate diagnostic quality. Stent implants caused negligible image artifacts.

CONCLUSIONS: Intra-aneurysmal contrast enhancement following endovascular treatment of intracranial aneurysms is a common finding and likely reflects the healing process. A long-term study to examine changes occurring with time and their association with packing density, type of coils (bare platinum versus bioactive coils), and aneurysm recurrence is underway (German Clinical Trials Register, DRKS-ID: DRKS00014644).

$\mathbf{T}$ he safety and efficacy of endovascular treatment of both ruptured and unruptured aneurysms have been shown in many large, multicenter trials. ${ }^{1-3}$ The main drawback of endovascular treatment is the recanalization of an initially occluded aneurysm. This occurs in about one-fourth to one-third of patients. In about $9 \%$ of patients, retreatment is required. ${ }^{4}$

Mechanisms leading to aneurysm recurrence include coil compaction secondary to poor packing or aneurysm growth ${ }^{5}$ and coil extrusion into the subarachnoid space or an intraluminal thrombus. ${ }^{6,7}$ Aneurysm recurrence is facilitated by poor thrombus organization due to the biologically inert platinum coil

Received November 14, 2019; accepted after revision December 30

From the Department of Neuroradiology, Faculty of Medicine, Medical Center-University of Freiburg, Freiburg, Germany.

Paper previously presented at: Annual Meeting of the German Society of Neuroradiology, October 3-6, 2018; Frankfurt, Germany.

This work was supported by the Research Committee, Albert-Ludwigs-Universität Freiburg.

Please address correspondence to Samer Elsheikh, MD, Klinik für Neuroradiologie, Breisacherstr 64, 79106 Freiburg, Germany; e-mail: samer.elsheikh@ uniklinik-freiburg.de

http://dx.doi.org/10.3174/ajnr.A6440 material; ${ }^{8}$ instability of the fresh, unorganized thrombus and degradation by thrombolysis; continued transmission of pulsations from the parent vessel into the aneurysm; ${ }^{9}$ incomplete neointimal coverage of the aneurysm neck; ${ }^{10}$ and neovessel formation in the lumen of the aneurysm, exposing the aneurysmal cavity to continuous blood flow. ${ }^{11}$

Following endovascular treatment of intracranial aneurysms, the aneurysms undergo a prolonged healing process. This process involves a cascade of events, including an inflammatory reaction occurring within the aneurysm and at its neck. This process may last $>1$ year. Ideally, aneurysm healing should end in a complete re-endothelialization of the aneurysm neck. ${ }^{11}$

Contrast enhancement following aneurysm coiling is a known observation, which is only rarely reported in the current literature. The 2 largest series were published by the same research group reporting their patient cohort. ${ }^{12,13}$

High-resolution T1-weighted contrast-enhanced MR blackblood imaging may depict not only enhancement of the vessel wall but also the sac contents. ${ }^{14}$ We hypothesized that contrast enhancement of the aneurysm wall and/or inside the intra-aneurysmal sac is associated with the healing process following 


\begin{tabular}{|c|c|c|c|}
\hline & 2D TIWI TIRM Black-Blood & 3D TIWI SPACE Black-Blood & 3D TIWI CS-SPACE Black-Blood \\
\hline Section orientation & Axial and coronal & Sagittal & Sagittal \\
\hline TR, TE, TI (ms), FA, NEX, PAT & $2000,11,1200,150^{\circ}, 2$, GRAPPA 2 & $\begin{array}{l}\text { 710, 11, N/A, N/A, 1, GRAPPA } 2 . \\
\text { echo train length } 157 \mathrm{~ms}\end{array}$ & $\begin{array}{l}800,10, \mathrm{~N} / \mathrm{A}, \mathrm{N} / \mathrm{A}, 1, \mathrm{GRAPPA} 2 . \\
\text { echo train length } 269 \mathrm{~ms}\end{array}$ \\
\hline FOV & $230 \times 201 \mathrm{~mm}$ & $230 \times 230 \mathrm{~mm}$ & $225 \times 225 \mathrm{~mm}$ \\
\hline Matrix & $512 \times 410$ & $256 \times 265$ & $448 \times 448$ \\
\hline Section thickness & $2 \mathrm{~mm}$ (10\% gap) & $0.9 \mathrm{~mm}$ & $0.64 \mathrm{~mm}$ \\
\hline Spatial resolution & $0.45 \times 0.45 \times 2 \mathrm{~mm}$ & $0.9 \mathrm{~mm}$ (isotropic) & $0.5 \times 0.5 \times 0.64 \mathrm{~mm}$ \\
\hline No. of slices & 15 & 192 & 256 \\
\hline Scan time & $4 \mathrm{~min} 6 \mathrm{sec}$ & $6 \mathrm{~min} 48 \mathrm{sec}$ & $6 \mathrm{~min} 54 \mathrm{sec}$ \\
\hline
\end{tabular}

Note:-FA indicates flip angle; TRIM, turbo inversion recovery magnitude; CS-SSPACE, sampling perfection with application-optimized contrasts by using different flip angle evolutions using compressed sensing; GRAPPA, generalized autocalibrating partially parallel acquisition; PAT, parallel acquisition technique.

aneurysm treatment, and as a corollary, contrast enhancement is associated with the stability of the result following aneurysm treatment. This study aims to describe the imaging findings of aneurysmal contrast enhancement on high-resolution 3D blackblood MR imaging following endovascular treatment and to assess the correlation between these findings and the presence of aneurysm recurrence and the elapsed time since coiling. Factors such as patient cooperation and image quality will also be addressed.

\section{MATERIALS AND METHODS \\ Research Design}

This was a nonrandomized, prospective, observational, diagnostic imaging study. The study was approved by the institutional review board. Informed written consent was obtained from all patients.

\section{Patients}

Consecutive patients, presenting for their regular follow-up MR imaging/MRA examination after endovascular treatment of a ruptured or unruptured intracranial aneurysm were included if they fulfilled the following criteria: 18 years of age or older and no known contraindication to gadolinium contrast agents (eg, previous moderate-to-severe allergic reaction or a glomerular filtration rate of $<60 \mathrm{~mL} / \mathrm{min} / 1.73 \mathrm{~m}^{2}$ ). No restriction on the time elapsed since the last endovascular treatment was imposed.

\section{MR Imaging Protocol}

The study was conducted using vendor-supplied sequences approved for clinical application. All examinations were performed on a Magnetom Prisma 3T scanner (Siemens, Erlangen, Germany) using a 64-element head coil.

Study sequences (Table 1) included pre- and postcontrast axial 2D, turbo inversion recovery magnitude, black-blood, fatsuppressed T1WI and sagittal 3D sampling perfection with application-optimized contrasts by using different flip angle evolution (SPACE sequence; Siemens) T1WI with and without compressed sensing. During the administration of the contrast medium, a coronal flash 3D and a time-resolved MR angiography sequence were acquired. Gadolinium contrast agent (gadoteridol, ProHance; Bracco Imaging S.p.A, Milan, Italy) was administered in a weight-adapted dose of $0.1 \mathrm{mmol} / \mathrm{kg}$ body weight intravenously.

\section{Study Sequences}

The spatial resolution and other scan parameters are summarized in Table 1.

\section{Assessment of Aneurysms and Black-Blood MR Imaging Sequences}

We determined the following measures:

- Presence and location of aneurysmal contrast enhancement: at the base (interface to the parent vessel), at the dome, inside the aneurysm sac, and in the sidewalls.

- Subjective evaluation of the aneurysmal contrast-enhancement intensity performed and graded using a 4-point semiquantitative scale: none/faint/moderate/strong.

- Analysis of the aneurysm size and location on the DSA and the interval between treatment and MRI examination.

- Image degradation due to artifacts after treatment using stents/flow diverters, subjectively determined.

- The rate of patient compliance for MRI-that is, the presence of motion artifacts and number of patients interrupting the examination.

\section{Aneurysm Recurrence}

The detection of aneurysm recurrence was based on the evaluation of images from nonenhanced TOF-MRA. It was defined as any visually detected signal inside an aneurysm of any size. Further DSA examinations were indicated only in the clinical context. The results of the DSA were not considered in this study.

\section{Statistical Methods}

Descriptive statistics were used for the demographic data of the patients. A 2-sided Fisher exact test was used to examine the correlation between aneurysm recurrence and the presence of contrast enhancement. An unpaired 2-sided $t$ test was used to assess the correlation between elapsed time since endovascular treatment and contrast enhancement.

\section{RESULTS}

Between April 2017 and February 2018, we prospectively included all consecutive patients presenting for the MR imaging follow-up examination after endovascular treatment of an intracranial aneurysm. A total of 31 patients ( 20 women) were included. One patient discontinued the examination before 
application of contrast medium. There were no observed adverse effects due to the administration of contrast medium.

\section{Patient and Aneurysm Data}

The demographic data of the patient cohort are summarized in Table 2. The different locations of the included aneurysms are summarized in Table 3.

Table 2: Patient demographic data

\begin{tabular}{lccc}
\hline & Median & Range & $\begin{array}{c}\text { Interquartile } \\
\text { Range }\end{array}$ \\
\hline Age $(\mathrm{yr})$ & 54 & $33-77$ & $47-61$ \\
Days since treatment & 417 & $87-5318$ & $189-1273$ \\
Aneurysm diameter $(\mathrm{cm})$ & 7 & $3-20$ & $5-10$ \\
\hline
\end{tabular}

Table 3: Location of intracranial aneurysms

\begin{tabular}{lcc}
\hline \multicolumn{1}{c}{ Aneurysm Location } & Frequency & Percentage \\
\hline Basilar artery, tip & 4 & $12.9 \%$ \\
Anterior cerebral artery, A1 segment & 1 & $3.2 \%$ \\
Anterior communicating artery & 12 & $38.7 \%$ \\
Internal carotid artery, paraophthalmic & 2 & $6.5 \%$ \\
Internal carotid artery, terminus & 1 & $3.2 \%$ \\
Anterior choroidal artery & 2 & $6.5 \%$ \\
Vertebral artery & 2 & $6.5 \%$ \\
Posterior communicating artery & 7 & $22.6 \%$ \\
\hline
\end{tabular}

Table 4: Association between aneurysm recurrence and aneurysmal contrast enhancement

\begin{tabular}{lccc}
\hline & \multicolumn{2}{c}{ Contrast Enhancement } & \\
\cline { 2 - 3 } Aneurysm Recurrence & Yes & No & Total \\
\hline Yes & 16 & 2 & 18 \\
No & 8 & 4 & 12 \\
Total & 24 & 6 & 30 \\
\hline
\end{tabular}

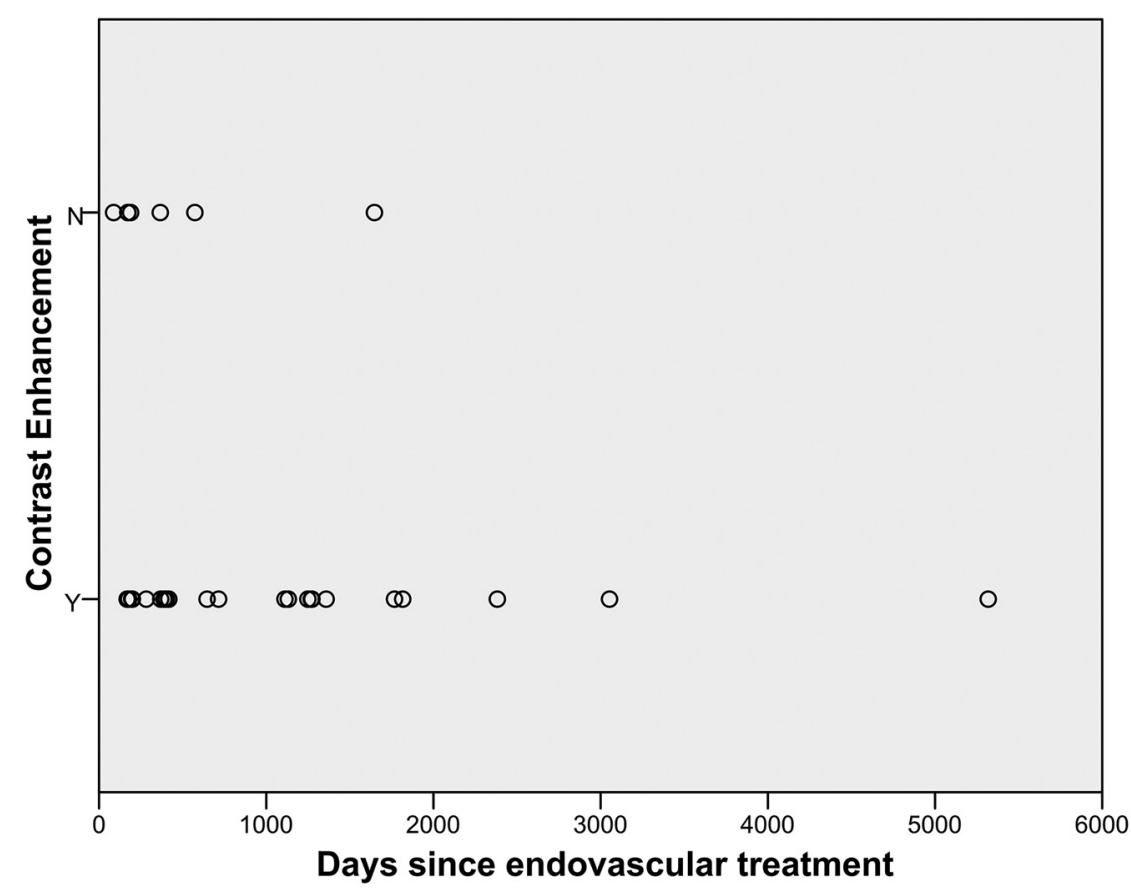

FIG 1. Temporal distribution of aneurysmal contrast enhancement analyzed in the days since endovascular treatment.

\section{Association between Aneurysm Recurrence and Contrast Enhancement}

The MR imaging-detected recurrence rate was $18 / 30$ (60\%). A 2-sided Fisher exact test (Table 4) showed no statistically significant association between aneurysm recurrence and aneurysmal enhancement $(P=.184)$.

\section{Elapsed Time since Coiling}

The median elapsed time since endovascular treatment was 417 days (interquartile range, 189-1273 days) (Table 2 and Fig 1). All aneurysms except one that did not show any contrast enhancement were imaged within 2 years after treatment. One aneurysm without contrast enhancement was examined at an interval of 4.5 years following treatment (Fig 1). Aneurysms with any contrast enhancement were imaged up to an interval of 14.5 years. An unpaired 2-sided $t$ test showed no statistically significant association between the elapsed time since endovascular treatment and contrast enhancement $(P=.83)$.

\section{Grading and Location of Aneurysmal Contrast Enhancement}

In 24/30 patients, contrast enhancement of the embolized aneurysm was observed. Eighteen aneurysms showed contrast enhancement solely in 1 location; 5 aneurysms, in 2 locations of the same aneurysm; and 1 aneurysm, in 3 different locations of the same aneurysm. Thus, a total of 31 locations of contrast enhancement in 24 aneurysms were recorded (Figs $2-4)$. Contrast enhancement inside the aneurysm sac was the most common location $(17 / 31,55 \%)$, followed by contrast enhancement of the sidewalls $(7 / 31,23 \%)$, and at the aneurysm base $(6 / 31,19 \%)$. Last, contrast enhancement of the aneurysm dome was observed in only 1 patient $(1 / 31,3 \%)$. Faint enhancement was observed 10/31 times, while moderate enhancement was observed 5/31 times. Strong enhancement was encountered 16/31 times. The results are listed in Table 5.

\section{Image Degradation/Artifacts}

Of 30 patients, 7 were treated using stents (flow diverter, $n=2$; stentassisted coiling, $n=3$; and bifurcation aneurysm stent, $n=2$ ). These metallic stent implants led to no or only minimal degradation of the image quality.

\section{Patient Compliance}

In $28 / 31$ patients, the images acquired were of good diagnostic quality. One patient discontinued the examination before the administration of contrast medium, so postcontrast images were not available for evaluation. Slight motion artifacts were observed in 2/31 patients. These artifacts were deemed to not greatly affect the diagnostic quality of the images and were included in our 


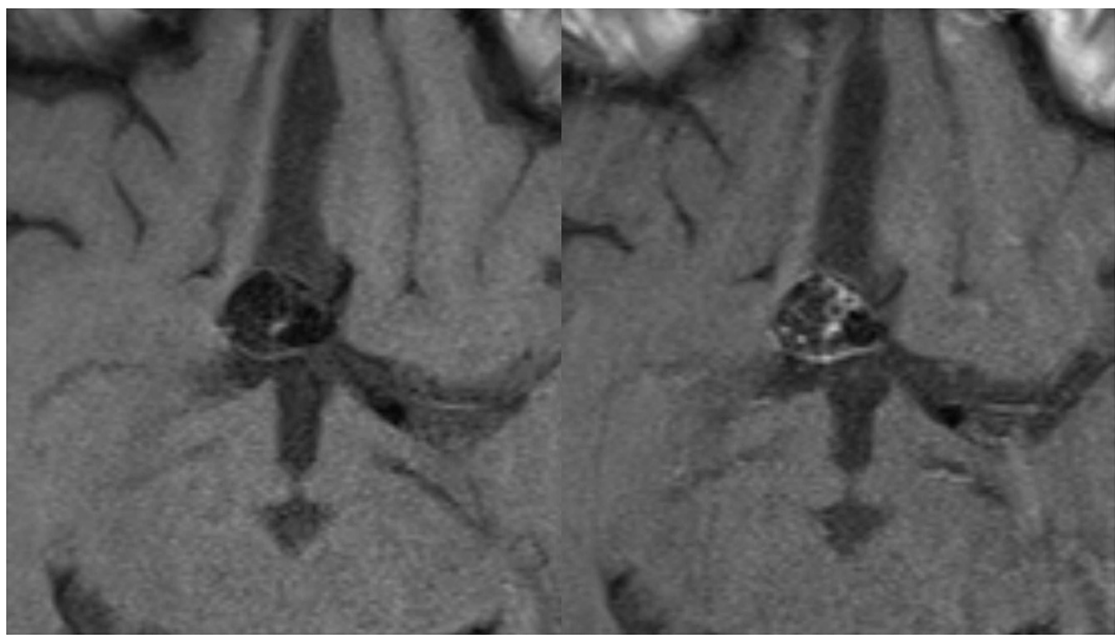

FIG 2. Anterior communicating artery aneurysm after endovascular treatment. Axial nonenhanced (left) and contrast-enhanced (right) black-blood MR images show enhancement of the sidewall and inside the aneurysm sac.

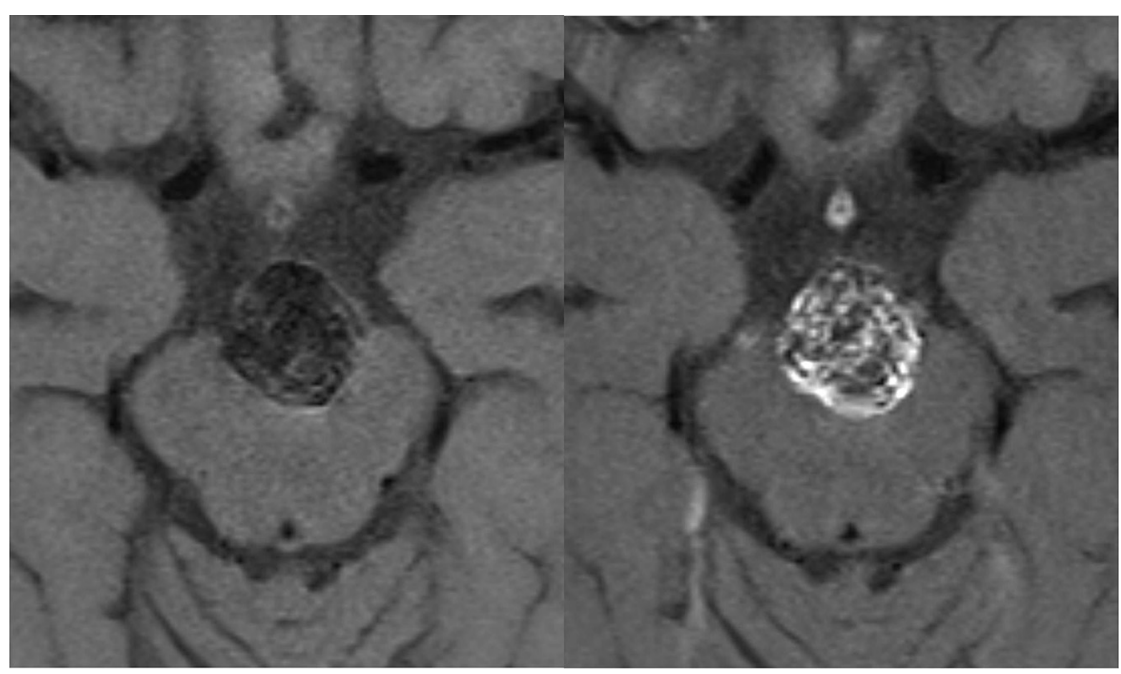

FIG 3. Tip of a basilar artery aneurysm after endovascular treatment. Axial nonenhanced (left) and contrast-enhanced (right) black-blood MR images show contrast enhancement of the sidewall and inside the aneurysm sac.

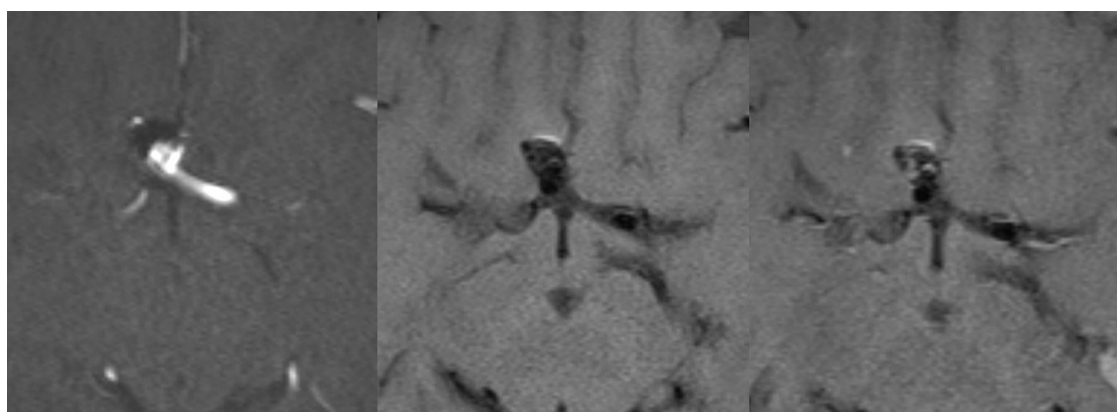

FIG 4. Anterior communicating artery aneurysm after endovascular treatment. Axial TOF-MRA (left) and axial nonenhanced (middle) and contrast-enhanced (right) black-blood MR images show recurrence at the base of the aneurysm. The same aneurysm shows contrast enhancement inside the aneurysm sac. evaluation. These 2 patients neither had a recurrence following endovascular treatment nor showed aneurysmal contrast enhancement on the study sequences.

\section{DISCUSSION}

The standard of care for follow-up after endovascular treatment of intracranial aneurysms includes regular MR imaging/MRA examinations. Our institutional standard is to perform follow-up MR imaging/MRA of intracranial aneurysms after endovascular treatment using 3T MR imaging and 3D TOF-MRA after 6, 18, 36, and 60 months. ${ }^{15}$ The decision to perform re-angiography and consider retreatment is made on the basis of the detection of relevant flow signal at the aneurysm base or within the aneurysm, compaction or expansion of the coil mass, and/or aneurysm growth.

In this study, we used contrastenhanced T1-weighted black-blood MR imaging sequences with a high spatial resolution at $3 \mathrm{~T}$. We used both axial and coronal 2D blackblood sequences with a high inplane resolution $(0.45 \times 0.45 \mathrm{~mm})$ at a section thickness of $2 \mathrm{~mm}$ and an isotropic 3D black-blood sequence (0.9 $\mathrm{mm}$ ). The higher signal-to-noise ratio of the $2 \mathrm{D}$ sequences allows a better assessment of the vessel wall and the sac content, and because the position of the aneurysm is known, the limited coverage of the $2 \mathrm{D}$ sequences is not disadvantageous. ${ }^{16}$ A future direction to further improve the spatial resolution of 3D black-blood imaging with whole-brain coverage at adequate scan times may be the use the recently introduced compressed sensing SPACE. ${ }^{17}$

The main result of our study was the detection of aneurysmal contrast enhancement in $80 \%(24 / 30)$ of the treated aneurysms. It occurred mostly within the aneurysm sac between the coils (55\%), but also in about $20 \%$ of cases in the aneurysm walls and/or at the aneurysm base. The etiology of the contrast-enhancing tissue is not clear; however, it is most likely related to organized blood clot, granulation tissue, and the subsequent healing process of the treated aneurysm. 
Table 5: Aneurysm enhancement location and grade in 24/31 aneurysms showing any enhancement

\begin{tabular}{|c|c|c|c|}
\hline $\begin{array}{l}\text { Case } \\
\text { No. }\end{array}$ & Aneurysm Location & $\begin{array}{l}\text { Location of Contrast } \\
\text { Enhancement }\end{array}$ & $\begin{array}{l}\text { Grading of Contrast } \\
\text { Enhancement }\end{array}$ \\
\hline 1 & AcomA & Inside the aneurysm & Moderate \\
\hline 2 & & Wall/sidewall & Strong \\
\hline 3 & Vertebral artery & Inside the aneurysm & Strong \\
\hline 4 & & Wall/sidewall & Strong \\
\hline 5 & Vertebral artery & Inside the aneurysm & Faint \\
\hline 6 & & Wall/sidewall & Faint \\
\hline 7 & PcomA & Base & Faint \\
\hline 8 & Basilar tip & Wall/sidewall & Strong \\
\hline 9 & Carotid bifurcation & Inside the aneurysm & Faint \\
\hline 10 & Paraophthalmic aneurysm & Inside the aneurysm & Faint \\
\hline 11 & AcomA & Inside the aneurysm & Strong \\
\hline 12 & AcomA & Base & Strong \\
\hline 13 & & Inside the aneurysm & Strong \\
\hline 14 & & Wall/sidewall & Strong \\
\hline 15 & Al segment & Base & Strong \\
\hline 16 & AcomA & Base & Moderate \\
\hline 17 & Anterior choroidal artery & Base & Strong \\
\hline 18 & PcomA & Inside the aneurysm & Strong \\
\hline 19 & PcomA & Inside the aneurysm & Faint \\
\hline 20 & Paraophthalmic aneurysm & Inside the aneurysm & Moderate \\
\hline 21 & PcomA & Inside the aneurysm & Faint \\
\hline 22 & Basilar tip & Inside the aneurysm & Faint \\
\hline 23 & Basilar tip & Base & Moderate \\
\hline 24 & PcomA & Inside the aneurysm & Strong \\
\hline 25 & AcomA & Inside the aneurysm & Strong \\
\hline 26 & & Wall/sidewall & Strong \\
\hline 27 & PcomA & Inside the aneurysm & Strong \\
\hline 28 & & Wall/sidewall & Strong \\
\hline 29 & AcomA & Inside the aneurysm & Faint \\
\hline 30 & AcomA & Inside the aneurysm & Faint \\
\hline 31 & AcomA & Dome & Moderate \\
\hline
\end{tabular}

Note:-AcomA indicates anterior communicating artery; PcomA, posterior communicating artery.

Most previously published studies using postgadolinium black-blood MR imaging sequences offered analysis of contrast enhancement in previously untreated, incidental intracranial aneurysms for potential use as an indicator of aneurysm instability or rupture risk ${ }^{18,19}$ or to define the site of an already ruptured aneurysm. $^{20,21}$ Literature regarding contrast-enhanced MR imaging of intracranial aneurysms following endovascular treatment is limited to examining patients using standard T1WI. ${ }^{12,13}$ Thus, to our knowledge, there are no published data systematically addressing contrast enhancement of intracranial aneurysms following endovascular treatment using black-blood MR imaging sequences.

We found no statistically significant association between contrast enhancement and the time elapsed since treatment. We also found no significant association with the presence of an aneurysm recurrence on the same MR imaging examination. A correlation between the presence and/or temporal changes of the contrast enhancement and the durability of the treatment result after endovascular aneurysm occlusion across time may be more interesting to examine, but these relationships are outside the scope of our study due to the cross-sectional nature of our data and the small sample size.

Overall, the image quality of treated aneurysms was adequate in our study. Images of patients treated with intravascular stent implants (including flow-diverter stents) showed only minimal artifacts without relevant image degradation. Guan et $\mathrm{al}^{22}$ reported similar findings regarding the utility of highresolution $3 \mathrm{D}$ black-blood $\mathrm{MR}$ imaging in the follow-up of intracranial aneurysms treated with flow diverters.

Limitations of our study are the small sample size and the cross-sectional nature of the data in our study cohort. Our cohort included patients with a wide range of intervals following the treatment of the aneurysms (up to about 14.5 years). Moreover, because we examined only aneurysms following endovascular treatment, there is a lack of histopathologic correlation of the treated aneurysms, so the exact cause of the contrast enhancement following treatment cannot be verified.

On the basis of this study, we started a long-term follow-up study to evaluate temporal changes of contrast enhancement during the follow-up period and to test for an association of contrast enhancement with aneurysm recurrence. This study is registered in the German Clinical Trials Register (DRKS-ID: DRKS00014644).

\section{CONCLUSIONS}

Contrast enhancement of intracranial aneurysms following endovascular treatment is a common finding of unknown clinical significance. It is mostly observed within the aneurysmal sac and likely is a sign of a healing process. We are currently running a long-term study to examine changes in aneurysmal contrast enhancement occurring across time and their association with aneurysm recurrence.

Disclosures: Samer Elsheikh-RELATED: Grant: Research Committee, Faculty of Medicine, University of Freiburg, Freiburg, Germany*; UNRELATED: Grants/ Grants Pending: Bracco SpA, Comments: research funding for another research article concerned with contrast enhancement of unrupruted aneurysms, not yet published.* Horst Urbach—UNRELATED: Board Membership: Co-Editor of Clinical Neuroradiology, Editorial Board of Neuroradiology, Comments: $1000 € /$ year; Payment for Lectures Including Service on Speakers Bureaus: Bayer AG, Bracco SpA, Stryker, UCB Pharma, Comments: less than 10,000 € since 2016; Other: shareholder of VEObrain $\mathrm{GmbH}$, Comments: no money paid. Stephan MeckelRELATED: Grant: Bracco SpA*; UNRELATED: Board Membership: Acandis GmbH, Comments: modest consultant's fee as member of the Scientific Advisory Board; Consultancy: Novartis Pharma GmbH, Comments: modest consultant's fee; Grants/Grants Pending: Novartis Pharma GmbH, Comments: MR imaging study grant*; Payment for Lectures Including Service on Speakers Bureaus: Medtronic, Comments: modest lecture fee; Payment for Development of Educational Presentations: Medtronic, Comments: money from Medtronic was paid to an individual; Travel/Accommodations/Meeting Expenses Unrelated to Activities Listed: Balt/AB Medica, MicroVention, Stryker.* *Money paid to the institution.

\section{REFERENCES}

1. Molyneux A, Kerr R; International Subarachnoid Aneurysm Trial Collaborative Group. International Subarachnoid Aneurysm Trial 
(ISAT) of neurosurgical clipping versus endovascular coiling in 2143 patients with ruptured intracranial aneurysms: a randomized trial. J Stroke Cerebrovasc Dis 2002;11:304-14 CrossRef

2. Pierot L, Cognard C, Ricolfi F, et al; CLARITY Investigators. Immediate anatomic results after the endovascular treatment of ruptured intracranial aneurysms: analysis in the CLARITY series. AJNR Am J Neuroradiol. 2010;31:907-11 CrossRef Medline

3. Pierot L, Spelle L, Vitry F, et al. Immediate clinical outcome of patients harboring unruptured intracranial aneurysms treated by endovascular approach: results of the ATENA study. Stroke 2008; 39:2497-504 CrossRef Medline

4. Naggara ON, White PM, Guilbert F, et al. Endovascular treatment of intracranial unruptured aneurysms: systematic review and meta-analysis of the literature on safety and efficacy. Radiology 2010;256:887-97 CrossRef Medline

5. Hope JK, Byrne JV, Molyneux AJ. Factors influencing successful angiographic occlusion of aneurysms treated by coil embolization. AJNR Am J Neuroradiol 1999;20:391-99 Medline

6. Ferns SP, van Rooij WJ, Sluzewski M, et al. Partially thrombosed intracranial aneurysms presenting with mass effect: long-term clinical and imaging follow-up after endovascular treatment. AJNR Am J Neuroradiol 2010;31:1197-205 CrossRef Medline

7. Waldron JS, Halbach VV, Lawton MT. Microsurgical management of incompletely coiled and recurrent aneurysms: trends, techniques, and observations on coil extrusion. Neurosurgery 2009;64: 301-15; discussion 315-17 CrossRef Medline

8. Yuki I, Lee D, Murayama Y, et al. Thrombus organization and healing in an experimental aneurysm model, Part II: the effect of various types of bioactive bioabsorbable polymeric coils. J Neurosurg 2007;107:109-20 CrossRef Medline

9. Boecher-Schwarz HG, Ringel K, Kopacz L, et al. Ex vivo study of the physical effect of coils on pressure and flow dynamics in experimental aneurysms. AJNR Am J Neuroradiol 2000;21:1532-36 Medline

10. Ozawa T, Tamatani S, Koike T, et al. Histological evaluation of endothelial reactions after endovascular coil embolization for intracranial aneurysm: clinical and experimental studies and review of the literature. Interv Neuroradiol 2003;9:69-82 CrossRef Medline

11. Brinjikji W, Kallmes DF, Kadirvel R. Mechanisms of healing in coiled intracranial aneurysms: a review of the literature. AJNR Am J Neuroradiol 2015;36:1216-22 CrossRef Medline
12. Fanning NF, Willinsky RA, ter Brugge KG. Wall enhancement, edema, and hydrocephalus after endovascular coil occlusion of intradural cerebral aneurysms. J Neurosurg 2008;108:1074-86 CrossRef Medline

13. Su IC, Willinsky RA, Fanning NF, et al. Aneurysmal wall enhancement and perianeurysmal edema after endovascular treatment of unruptured cerebral aneurysms. Neuroradiology 2014;56:487-95 CrossRef Medline

14. Tan HW, Chen X, Maingard J, et al. Intracranial vessel wall imaging with magnetic resonance imaging: current techniques and applications. World Neurosurg 2018;112:186-98 CrossRef Medline

15. Urbach H, Dorenbeck $U$, von Falkenhausen $M$, et al. Three-dimensional time-of-flight $\mathrm{MR}$ angiography at $3 \mathrm{~T}$ compared to digital subtraction angiography in the follow-up of ruptured and coiled intracranial aneurysms: a prospective study. Neuroradiology 2008; 50:383-89 CrossRef Medline

16. Eiden S, Beck C, Venhoff $\mathrm{N}$, et al. High-resolution contrastenhanced vessel wall imaging in patients with suspected cerebral vasculitis: prospective comparison of whole-brain 3D T1 SPACE versus 2D T1 black-blood MRI at 3 Tesla. PLoS One 2019;14: e0213514 CrossRef Medline

17. Zhu C, Tian B, Chen L, et al. Accelerated whole brain intracranial vessel wall imaging using black blood fast spin echo with compressed sensing (CS-SPACE). MAGMA 2018;31:457-67 CrossRef Medline

18. Etminan N, Rinkel GJ. Unruptured intracranial aneurysms: development, rupture and preventive management. Nat Rev Neurol 2016;12:699-713 CrossRef Medline

19. Hackenberg KA, Hanggi D, Etminan N. Unruptured intracranial aneurysms. Stroke 2018;49:2268-75 CrossRef Medline

20. Matouk CC, Mandell DM, Gunel M, et al. Vessel wall magnetic resonance imaging identifies the site of rupture in patients with multiple intracranial aneurysms: proof of principle. Neurosurgery 2013; 72:492-96; discussion 496 CrossRef Medline

21. Nagahata $S$, Nagahata $M$, Obara $M$, et al. Wall enhancement of the intracranial aneurysms revealed by magnetic resonance vessel wall imaging using three-dimensional turbo spin-echo sequence with motion-sensitized driven-equilibrium: a sign of ruptured aneurysm? Clin Neuroradiol 2016;26:277-83 CrossRef Medline

22. Guan J, Karsy M, McNally S, et al. High-resolution magnetic resonance imaging of intracranial aneurysms treated by flow diversion. Interdiscip Neurosurg 2017;10:69-74 CrossRef 